\title{
Words Matter
}

\author{
Jodi B. Segal, MD, MPH and Nancy L. Schoenborn, MD, MHS
}

Johns Hopkins University School of Medicine, Baltimore, MD, USA.

KEY WORDS: communication; overuse; patient preference.

J Gen Intern Med 33(10):1607-9

DOI: $10.1007 / \mathrm{s} 11606-018-4523-6$

(c) Society of General Internal Medicine 2018

\section{INTRODUCTION}

Choosing Wisely aims to promote conversations between clinicians and patients that will help patients to choose care that is "supported by evidence, not duplicative of other tests or procedures already received, free from harm, and truly necessary." With the goal of improving how patients and doctors communicate on these topics, we sought to understand what words resonate most with the public to describe use of services where the likelihood of harm exceeds the likelihood of benefit. ${ }^{2}$

\section{METHODS}

As part of an online survey about cancer screening, we queried individuals 65 years and older from the KnowledgePanel, a product of Knowledge Networks. ${ }^{3}$ KnowledgePanel is a probability-based web panel with more than 55,000 members that is designed to be representative of the US adult population. Panelists are sent emails three to four times a month inviting them to participate in online surveys. Participants responded to our invitation and were then randomized to respond to one of two clinical scenarios. One scenario described use of plain film $x$-rays for subacute low back pain in an older person without worrisome symptoms; the other described the use of antibiotics in a healthy, non-smoker with a chest cold. We chose these two examples because they have been named by multiple professional societies as services where harms from use outweigh the benefits. The participants were then further randomized to answer whether the described service was or was not (1) "inappropriate," (2) "wasteful," (3) "medically unnecessary," or an (4) "overused test/treatment." Each participant saw only one of these four phrases. We also asked, when answering the question, whether they considered the cost and harms of the test or treatment.

\section{RESULTS}

Of the 1272 panel members asked to respond, 881 (69.3\%) completed the questions in November 2016. Survey weights were used to adjust for nonresponse and oversampling of African Americans. The mean age of participants was 73.4 years of whom $55 \%$ were women. In the low back pain scenario, despite our stating that $\mathrm{x}$-ray is not considered to be informative, most participants did not classify the test as being "inappropriate" or "wasteful." A significant majority (63\%), however, did agree that the x-ray was "medically unnecessary," and slightly more than half thought the test was "overused" in this scenario (Fig. 1). In the antibiotic scenario, the term "overused treatment" resonated most with the participants with $71 \%$ agreeing that this describes the usage portrayed in the scenario. Only a slight majority thought that the antibiotic use was "inappropriate," "wasteful," or "medically unnecessary" (Fig. 2).

Only $34 \%$ of the participants said that they took cost into consideration in responding to the question about the x-ray scenario, with 5\% not responding. Participants who agreed that the test was unwarranted were slightly more likely than not to say that cost was a consideration when presented with the terms "wasteful" or "medically unnecessary." This was not so for the people seeing the words "inappropriate" or "overused test." Forty-seven percent of participants considered the harms of x-ray when responding. The harms particularly motivated those who affirmed that the x-ray is an "overused test." In the antibiotic scenario, only $9 \%$ of participants took the costs of the antibiotic into consideration, while $68 \%$ considered the harms of antibiotics when responding. The majority of participants who agreed that the antibiotic was unwarranted said they were motivated by harms - regardless of which phrase they saw.

\section{DISCUSSION}

We conclude that words matter. The precise word choice we use to discuss interventions where the harms outweigh the benefit may affect patient understanding, perception, and subsequent acceptance of the recommendations. The

Published online June 8, 2018 


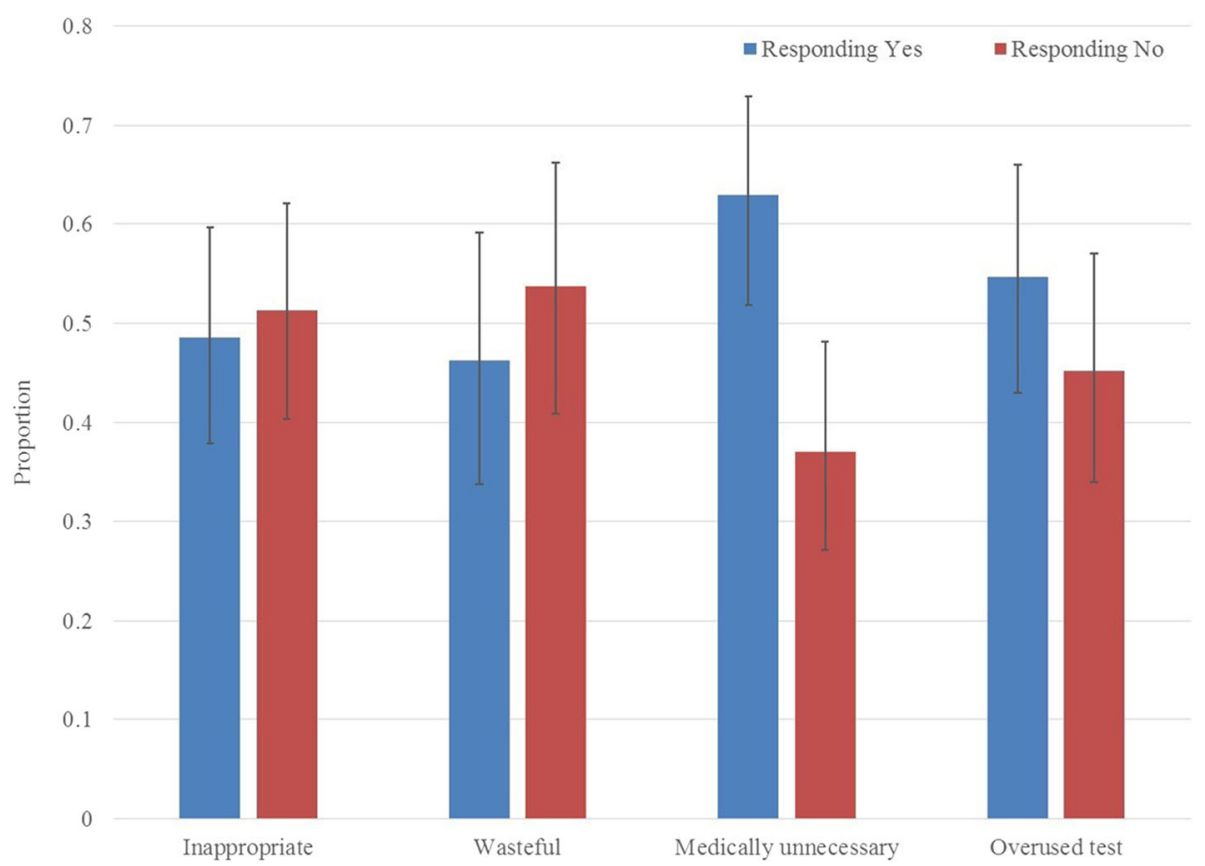

Figure 1 Responses to different phrases describing the X-ray scenario $(n=469)$.

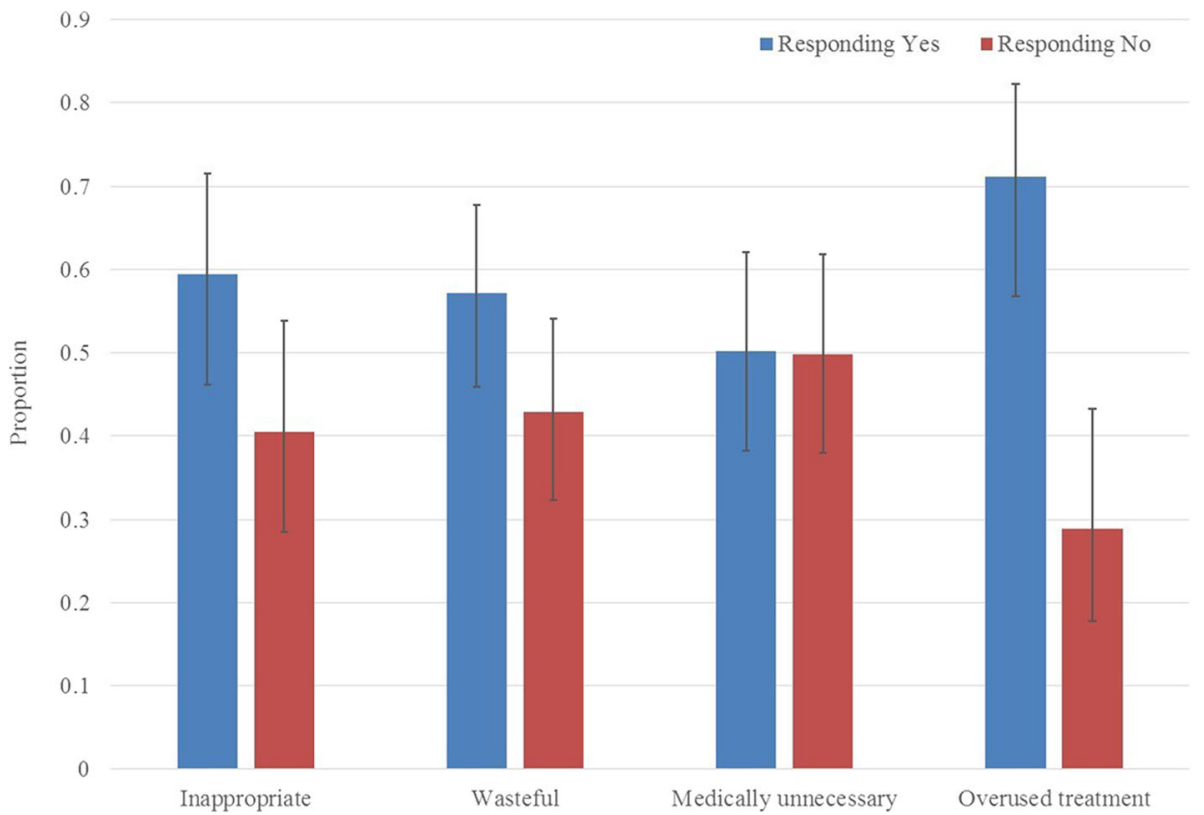

Figure 2 Responses to different phrases describing the antibiotic scenario $(n=412)$.

preferred word choice in our study to describe interventions where harms outweigh the benefits differed depending on if diagnostic test or treatment was involved. Results suggest that phrases with more clinical association, such as "medically unnecessary," may be more preferred than other commonly used terms. Limitations of the study include focus on older adults and use of hypothetical scenarios. Further research is needed to better understand why the responses differed importantly across these scenarios.
Corresponding Author: Nancy L. Schoenborn, MD, MHS; Johns Hopkins University School of Medicine, Baltimore, MD, USA (e-mail: nancyli@jhmi.edu).

Funding/Support Research reported in this publication was supported by the National Institute On Aging of the National Institutes of Health under Award Number R03AG050912. The content is solely the responsibility of the authors and does not necessarily represent the official views of the National Institutes of Health.

In addition, Dr. Schoenborn was supported by a T. Franklin Williams Scholarship Award with funding provided by the Atlantic 
Philanthropies, Inc., the John A. Hartford Foundation, the Alliance for Academic Internal Medicine-Association of Specialty Professors, and the American Geriatrics Society; the Johns Hopkins KL2 Clinical Scholars program funded by KL2TR001077 from the National Center for Advancing Translational Sciences (NCATS), a component of the National Institutes of Health (NIH), and NIH Roadmap for Medical Research; and a Cancer Control Career Development Award from the American Cancer Society (CCCDA-16-002-01). Dr. Segal was supported by K24AG049036 from the National Institute on Aging.

The funding sources had no role in the design, methods, subject recruitment, data collections, analysis, and preparation of paper.

\section{Compliance with Ethical Standards:}

Conflict of Interest: The authors declare that they do not have a conflict of interest.

\section{REFERENCES}

1. Cassel CK, Guest JA. Choosing wisely: Helping physicians and patients make smart decisions about their care. JAMA. 2012;307(17):1801-1802

2. Agency for Healthcare Research and Quality. Glossary: underuse, overuse, misuse. https://psnet.ahrq.gov/glossary/u. Accessed 5/2/18.

3. GfK Knowledge Networks. http://www.knowledgenetworks.com/ganp/. 2017 GfK Custom Research North America. Accessed 5/2/18. 